\title{
EL PROGRESIVO ENCANTO POR LA GESTIÓN PRIVADA: ANÁLISIS DE LOS MODELOS DE LA GESTIÓN PÚBLICA-PRIVADA EN ENSEÑANZA MEDIA EN URUGUAY (2002-2013)
}

\author{
Eloísa Bordoli ${ }^{1}$ \\ STEFANÍA CONDE ${ }^{2}$
}

\begin{abstract}
RESUMEN: El objetivo del artículo fue analizar tres experiencias de los modelos de gestión pública-privada en la enseñanza media, desarrolladas en las últimas décadas en Uruguay. Particularmente se presentarán el Programa Aulas Comunitarias y los liceos Jubilar e Impulso. El primer programa constituye una experiencia particular, en tanto articula una gestión compartida entre el Consejo de Educación Secundaria y las diversas Organizaciones de la Sociedad Civil. Los otros dos liceos se inscriben en los modelos de gestión privada con financiamiento público, característicos de las formas de privatización en el campo educativo. Interesa especialmente contextualizar dichas experiencias en el marco de la disputa en el campo simbólico en torno al papel que el Estado, las Organizaciones de la Sociedad Civil y las empresas ocupan en el ámbito de la educación pública. Asimismo importa identificar el proceso de retracción de la educación como un derecho universal y el surgimiento y progresivo asentamiento de la educación como un producto susceptible de ser comercializado que se está produciendo en las últimas décadas en Uruguay. Se propuso realizar el análisis en torno a tres ejes: contextualizar y analizar la configuración que el valor de lo público ha tenido en el sistema educativo uruguayo; ubicar el contexto de surgimiento y las características de cada una de las experiencias educativas señaladas, apreciando las particularidades que asumen estas modalidades; analizar las tensiones y las disputas simbólicas en torno a las vinculaciones entre lo público y lo privado así como las resignificaciones del sentido de la educación pública, con los riesgos que ello comporta.
\end{abstract}

Palabras clave: Educación pública. Derecho. Procesos privatizadores.

\footnotetext{
${ }^{1}$ Universidad de la República, Facultad de Humanidades y Ciencias de la Educación - Montevideo, Uruguay. E-mail: eloisabordoli@gmail.com

${ }^{2}$ Universidad de la República, Instituto de Educación de la Facultad de Humanidades y Ciencias de la Educación - Montevideo, Uruguay. E-mail: stefa07_87@hotmail.com

DOI: 10.1590/ES0101-73302016157845
} 


\section{THE PROGRESSIVE CAPTIVATION OF PRIVATE MANAGEMENT: ANALYSIS OF PUBLIC-PRIVATE MANAGEMENT MODELS IN MIDDLE EDUCATION IN URUGUAY (2002-2013)}

ABSTRACT: The aim of this article was to analyze three experiences of public-private management models in middle education that were developed in the last decades in Uruguay. In particular, the Programa de Aulas Comunitarias and the high schools Jubilar and Impulso will be presented. The first program constitutes a special experience, because it articulates a mixed management between the Consejo de Educación Secundaria and various Organizations of the Civil Society. The other two programs regard private management models with public financing, which are characteristic of privatization forms in the education field. There is a special interest in contextualizing these experiences within the framework of the dispute going on in the symbolic field regarding the role that State, Civic Society organizations and companies have in public education. In addition, there is a concern in identifying the retraction process of education as a universal right and its rise and progressive settlement as a product prone to be commercialized that has been taking place in Uruguay in the last decades. This analysis was proposed to be carried out around three axes: contextualize and analyze the value public has had within the Uruguayan education system; identify the context in which each of the three experiences arise and their characteristics, considering the peculiarities of each program; and analyze the symbolic tensions and disputes taking place around the relations between public and private activities, as well as the new meaning of the public education sense, with the risks it implicates.

Keywords: Public education. Right. Privatization processes.

\section{LE PROGRESSIF ENCHANTEMENT POUR LA GESTION PRIVÉE: ANALYSE DE MODÈLES DE GESTION PUBLIC-PRIVÉ DANS L'ENSEIGNEMENT SECONDAIRE EN URUGUAY (2002 - 2013)}

RÉSUMÉ: Le but de l'article est d'analyser trois expériences de modèles de gestion public-privé dans l'enseignement secondaire développpés au cours des dernières décennies en Uruguay. Particulièrement on présentera le Programme Salles Communautaires et les lycées Jubilar et Impulso. Le premier Programme constitue une expérience particulière du moment qu'il fait l'articulation d'une gestion partagée par le Conseil d'Éducation Secondaire et diverses Organisations de la Société Civile. Les deux lycées cités sont compris dans le modèle de gestion privée avec financement public, qui sont caractéristiques des formes de privatisation dans le champ éducatif. Il nous intéresse spécialement de mettre en contexte ces expériences dans le cadre de la dispute sur le champ symbolique autour du rôle que l'État, les Organisations de la Société Civile et les entreprises jouent dans le domaine de l'éducation publique.Il est important également d'identifier le processus qui s'est développé dans les dernières décennies 
en Uruguay de rétraction de l'éducation en tant que droit universel et d'apparition et progressif établissement de l'éducation comme produit susceptible d'être commercialisé. On se propose l'analyse autour de trois axes: la mise em contexte et l'analyse de la configuration que la valeur de ce qui est public a eu dans le système éducatif uruguayen, la situation du contexte où font l'apparition et les caractéristiques de chacune des expériences éducatives identifiées, et en apprécier leurs particularités singulières, l'analyse des tensions et des disputes symboliques autour des rapports public-privé ainsi que de nouvelles significations du sens de l'éducation publique y compris les risques que cela comporte.

Mots-clés: Éducation publique. Droit. Processus de privatisation.

\section{Introducción}

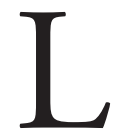

os nuevos modos de privatización y mercantilización de la educación que se están procesando a nivel mundial, y particularmente en América Latina, se articulan con tres procesos de orden general: la crisis del capitalismo, la progresiva hegemonía de la ideología globalizadora y las formas de reconfiguración neoliberal (LEHER, 2009). Estos procesos no sólo ofrecen un continente a las nuevas modalidades de presentar lo educativo en sociedades latinoamericanas signadas por la fragmentación y la desigualdad, sino que también brindan el contenido significante que las justifica y las dota de sentido. De esta manera, las mutaciones de lo público como valor y de la educación pública como derecho ciudadano y deber del Estado presentan rasgos "multifacéticos" (GENTILI; STUBRIN, 2009, p. 9), los cuales, muchas veces, enmascaran las alteraciones y las restricciones al Estado que las dinámicas globales y privatizadoras articulan.

Es preciso subrayar que estos procesos se operan en el marco de luchas por: resistir las restricciones y ampliar los espacios de lo común acaecidos en el espacio simbólico y socio-histórico. Esos debates se inscriben en procesos históricos particulares y contradictorios en los que se amplía el creciente ingreso a la educación pública de sectores largamente excluidos de este derecho, al tiempo que se habilita la implementación de crecientes procesos de privatización. Esta paradoja parecería constituirse en uno de los rasgos de nuestro tiempo.

Concomitantemente a estos procesos generales, se aprecia cómo las formas escolares modernas que articularon una particular manera de entender lo público, se presentan "desfasadas" con respecto a las demandas sociales. La forma escolar, en cuyo horizonte utópico inscribía a omnes et singulatim, se halla en crisis ante estas nuevas demandas. 
En este entramado, el Uruguay moderno y el sistema educativo se configuraron como garantes de una sociedad que inscribía su sueño utópico en una educación pública integradora e igualitaria. En este sentido, esta marca constitutiva actualmente es doblemente interpelada por: el ingreso al sistema educativo de nuevos sectores que estaban al margen del mismo y por los procesos privatizadores que procuran restringir lo público. La primera de estas interpelaciones pone en cuestión la forma escolar (arreglos institucionales y dinámicas curriculares), especialmente en relación a los sectores en situación de pobreza. El segundo elemento se refiere a la lucha ideológica y a las maneras socio-históricas de articulación de lo escolar respecto de la sociedad, al Estado y al sector empresarial.

En estas líneas interesa presentar la manera en que en Uruguay se fue constituyendo y articulando la escuela en el espacio público, su valor y su relación con el Estado. Asimismo, se analizaron los modos de representar los elementos que dan cuenta de una crisis de lo escolar en el marco de los procesos de reforma neoliberal de los 1990, además de las tensiones que se articulan en torno a lo público en el campo educativo.

\section{El valor de lo público en el origen del sistema educativo uruguayo}

Este apartado desarrolla dos hipótesis: la escuela pública se configuró en el proyecto del Estado moderno al tiempo que ésta consolidó al Estado, y el horizonte utópico del proyecto de una sociedad de iguales e integradora requirió la consolidación de la escuela pública como un derecho ciudadano y deber del Estado.

Es posible concebir a la escuela como una de las mayores construcciones de la modernidad. Como señalan Pineau et al. (2001), "escuela y modernidad establecieron una relación de producción mutua, siendo la escuela fundida en el paisaje moderno, presentándose así desde una condición 'natural'”. En este contexto, se destaca la función social de la educación, en tanto constructora de igualdad a nivel social. El principio de igualdad fue uno de los pilares de la expansión de los sistemas educativos, tornándose un sinónimo de homogeneidad. En Uruguay, el sistema educativo se configuró a partir de la Reforma Vareliana ${ }^{1}$, proceso que coadyuvó a la consolidación del Estado uruguayo en el marco del cual la educación se presentó como base de la ciudadanía y pilar de la República, destacándose su papel integrador, concebida como acto político, en tanto vehículo para la construcción de la Nación.

La propuesta vareliana se dirigía a que todos fueran socializados según un patrón único, siendo la condición para el logro de la igualdad: 
Gratuita, para todos, abierta a todos, recibiendo en sus bancos niños de todas las clases y de todos los cultos, hace olvidar las disensiones sociales, amortigua las animosidades religiosas, destruye las preocupaciones y las antipatías, e inspira a cada uno el amor de la patria común y el respeto por las instituciones libres. [...] Los que una vez se han sentado juntos en los bancos de una escuela, en la que eran iguales, a la que concurrían usando de un mismo derecho, se acostumbran fácilmente a considerarse iguales, a no reconocer más diferencias que las que resultan de las aptitudes y las virtudes de cada uno: y así, la escuela gratuita es el más poderoso instrumento para la práctica de la igualdad democrática. (VARELA, 1973, p. 56).

Este proyecto se articuló en torno a los principios de 'laicidad', 'gratuidad' y 'obligatoriedad' con la intencionalidad de que la escuela pública, al amparo del Estado, albergara al conjunto de ciudadanos en una sociedad que estaba signada por la diversidad cultural, la desigualdad social y las luchas político-partidarias. Desde este lugar, al decir de Bordoli (2006), es posible establecer una equivalencia discursiva entre educación pública e integración social, a la vez que entre igualdad y homogeneización. Igualdad y educación se implican una a la otra, configurándose así una unidad de sentido. La educación constituía el vehículo privilegiado para el logro de la integración y el desarrollo de la sociedad.

Por lo tanto, todos debían ser socializados según un patrón único, más allá de cualquier diferencia. Como señala Dussel (2004), la escolaridad se concibió como un terreno neutro y universal, que abrazaría por igual a todos los habitantes. De este modo,

Si esta identidad igualitaria se definía no sólo por la abstracción legal de nivelar y equiparar a todos los ciudadanos sino también porque todos se condujeran de la misma manera, hablaran el mismo lenguaje, celebraran a los mismos héroes y aprendieran las mismas cosas, entonces quienes persistieran en afirmar su diversidad serían percibidos como un peligro para esta identidad colectiva, o como sujetos inferiores que aún no habían alcanzado el mismo grado de civilización. (DUSSEL, 2004, p. 1).

La escuela fue concebida como un espacio físico y simbólico que habilitaría un lugar de encuentro entre diversos sujetos, siendo posible la inclusión en un "nosotros social". Es en este marco que asistimos a una "utopía educacionista' que "[...] se desarrolla sobre la base de aspirar a que la educación colme, constituya plenamente a los sujetos, convirtiéndolos en ciudadanos" (BORDOLI; MARTINIS, 2010). Por lo tanto, advertimos el carácter universalista e igualita- 
rio propio del origen del sistema educativo. Cabe destacar la configuración de la educación como un derecho, en tanto acceso al patrimonio cultural, indispensable para el logro de la integración y progreso social. En este sentido el discurso escolar moderno se calificó a sí mismo como democrático y democratizador.

La escolarización fue una invención de la modernidad, contexto en el que surge la Escuela, como una institución pública, durante el marco de la alianza fundacional (CASSASUS, 1995) entre Estado y Educación. Es como resultado de esta alianza política que la educación se convierte en función pública y en proyecto nacional, instaurándose los sistemas educativos. Hay un proyecto político con un claro objetivo civilizatorio.

\section{La 'crisis educativa' y la 'crisis del valor de lo público"}

En el período post-dictadura ${ }^{2}$ en Uruguay, particularmente a partir de la década de los 1990, se apreció la articulación de un discurso que reconoce las dificultades del sistema educativo para dar cabida a los sectores históricamente postergados de la sociedad "culpabilizando" al Estado por estas dificultades. En este marco, se suele afirmar y reconocer que se asiste a una "crisis educativa" de la educación media en particular ${ }^{3}$ —, la que ha ganado centralidad en la agenda pública debido a las problemáticas para que los sujetos permanezcan y finalicen sus estudios en la educación formal.

La disconformidad social con el desempeño del sistema educativo uruguayo ha renovado las discusiones sobre su arquitectura y anclaje institucional. Es posible referirse a una crisis orgánica de los sistemas educativos modernos, a los límites del proyecto educativo integracionista moderno basado en el cuestionamiento al ethos igualitario. Como principales expresiones sobre las que se asienta esta crisis se puede mencionar: el fracaso escolar, los bajos resultados de las evaluaciones estudiantiles y la desafiliación de los sujetos del sistema educativo. La deserción se presenta como el principal problema educativo, asociado en la educación media a dos factores: la trayectoria educativa inicial y el medio de origen del estudiante (ARISTIMUÑO, 2009; FERNÁNDEZ, 2010). Ante estas cuestiones, la región ha implementado reformas en materia educativa.

A los problemas reseñados, se debe anexar una constelación de nociones: globalización, competitividad, agencias de préstamo, etc. (FRIGERIO, 2000) que ofrecen la configuración de nuevos anudamientos de sentido y tensionan el ethos igualitario fundante del sistema educativo. Desde la articulación de los elementos críticos y estas nociones, las reformas de los 1990 habilitaron los procesos privatizadores $^{4}$. Los hilos argumentales que procuran suturar elementos de una crisis de la educación media con el declive de lo público y de las formas de gestión privada son impulsados por los sectores neoliberales en Uruguay, que encontraron fuertes 
resistencias en los gremios y partidos políticos de la oposición en ese período 5 . No obstante ello, en el universo simbólico se instala progresivamente una constelación de sentidos que asocian a lo público con lo ineficiente y a lo privado con lo eficiente y eficaz. Esto significa que a nivel de la configuración de los "universos de sentidos" (PÊCHEUX, 1990) y de la lucha simbólica por hegemonizarlos, se articula un discurso que hace foco en el debilitamiento de lo público y en los 'beneficios' de lo privado que trasciende los sectores políticos neoliberales y se asienta en el 'sentido común'.

En el marco de la matriz constitutiva de la escuela pública uruguaya y por las resistencias de sectores sindicales y sociales a las privatizaciones es que la reforma de los 1990 en Uruguay adquiere rasgos diferenciales a los de la región. En este sentido, se podría plantear que en este país se operaron "reformas liberales amortiguadas" (BENTANCUR, 2008, p. 230), básicamente por cuatro factores explicativos:

- la matriz histórica configurada en torno al valor de lo público (como desarrollamos precedentemente);

- el carácter gradualista que la cultura política uruguaya ha presentado en el siglo XX;

- la política pluralista caracterizada por un cierto equilibrio de fuerzas entre los sectores gobernantes y de la oposición (LANZARO, 2004); y

- la centralidad y resistencia de actores sociales (sindicatos, asociación de jubilados, etc.) ante los cambios y la posibilidad constitucional de democracia directa de la ciudadanía - que, en usufructo de ella - derogó las reformas más neoconservadoras del período (MOREIRA, 2001).

El interjuego de los cuatro elementos provocó que, en este período, la reforma educativa tuviera rasgos heterodoxos y amortiguados. Las políticas de los 1990 en educación impulsaron políticas focalizadas y de corte asistencial hilvanando una constelación de sentidos que ubican a la educación pública como educación para los pobres. Estas construcciones de sentido estuvieron asociadas a "procesos de gobierno de poblaciones en situación de pobreza que a preocupaciones estrictamente educativas" (MARTINIS, 2013, p. 118). Podemos señalar que en las políticas educativas vigentes en los 1990 se configura una triple construcción discursiva:

- la concepción del sujeto de la educación como niño carente;

- planteo de un modelo escolar para atender a estos nińos, en el marco del cual la educación fue ubicada dentro de políticas sociales, negándose así su función principal, o sea la transmisión del patrimonio cultural; 
- la concepción de los maestros como técnicos, asistentes sociales o contenedores, en detrimento de su papel de trasmisor de cultura y saberes considerados relevantes por la sociedad.

Desde este lugar, se renunció a la posibilidad de la educación, anulando al sujeto alumno que devino "carente" e imposibilitado de aprender. La categoría que adquirió fuerza en detrimento de la igualdad fue la de equidad, de modo que las políticas procuraban desarrollar acciones para igualar a los sujetos en el punto de partida, siendo la desigualdad un dato a priori a constatar en la realidad. En esta línea:

La naturalización de la desigualdad ubica a las políticas que la hacen posible como políticas de la producción de la injusticia $[. .$.$] . Se produce un proceso de fragmentación social que$ tiene su correlato en el sistema educativo, el cual también sufre una situación de fragmentación. Una política educativa o una intervención educativa puntual que sea concebida desde esta perspectiva niega la posibilidad de la educación en tanto concibe al otro desde su carencia o desde su peligrosidad. Lo concibe desde lo que no tiene y prefija un destino, anticipa un futuro clausurando la posibilidad de acontecimiento de lo nuevo. (MARTINIS, 2006, p. 3).

La familia (particularmente considerando el estado conyugal de los padres y el nivel de instrucción de la madre) fue visualizada como responsable del fracaso escolar, a la vez que el medio social fue un obstáculo para la acción educativa. Surge así la referencia al "contexto" con un significado de carencia, pobreza y marginalidad. Se lo ubica como el "afuera" de la institución que amenaza.

Actualmente, se ha generalizado y fortalecido el diagnóstico de la ineficiencia de la educación pública asociada a los resultados. Al respecto:

[...] tampoco es posible desconocer que va en aumento en la opinión pública un discurso que demoniza a la educación pública mostrándola como atrasada e ineficiente, basando sus elaboraciones, por ejemplo, en los resultados del país en la prueba internacional PISA. Estas formas de ataque a la educación pública encuentran amplios ecos en importantes medios de comunicación del país, los cuales cotidianamente insisten en la supuesta caducidad del modelo educativo, reclamando la incorporación de formas de financiamiento estatal a la educación privada y la incorporación de formas de gestión empresarial en la educación pública. (MARTINIS, 2012, en GIROUX, 2012). 
En síntesis, en Uruguay, las reformas del Estado y educativa tuvieron un carácter heterodoxo o amortiguado por las resistencias de las organizaciones sindicales y características socio-históricas reseñadas. No obstante, en el universo de la representación simbólica, se comienza a instalar, en forma moderada pero progresiva, una asociación discursiva caracterizada por el desvalor de lo público y el progresivo encanto de la "eficiencia" privada. Estas discursividades no han logrado estabilizarse sino que se hallan en una zona de disputa con las que reivindican el valor de lo público y el rol central del Estado.

\section{Nuevo gobierno con cambio de signo}

En la última década el mapa político del Uruguay se modificó al asumir, por primera vez en la historia, un gobierno de centro-izquierda ${ }^{6}$, el Frente Amplio (FA). En relación a las características de este primer gobierno del FA (20052010), es posible afirmar que presenta una tendencia de "izquierda gradualista y racional" (MOREIRA et al., 2008, p. 15) o socialdemócrata (MAYORGA, 2009). $\mathrm{Al}$ igual que los otros gobiernos de América Latina, el del FA articula su discurso crítico sobre la herencia "maldita" del neoliberalismo, de los efectos económicos desbastadores de los ajustes estructurales operados en los 1990 y de las graves consecuencias sociales que los mismos ocasionaron. Este discurso crítico se enfrenta a los avances gradualistas o heterodoxos, pero "reales", del capital y el mercado sobre el Estado y sus efectos en la sociedad y la política así como la configuración discursiva que entrona al mercado como modelo y pensamiento único.

De este modo, el discurso del FA coincide con las características generales de los nuevos gobiernos progresistas (NGP) de la región, los cuales presentan:

un denominador común político [...] caracterizado por una relativa alta oposición al consenso político reformista de los noventa, la adscripción a ideas y programas políticos tendientes a recomponer algunos de los más agudos efectos sociales, políticos e institucionales generados por aquellas políticas, y un giro ideológico más heterogéneo [...] (MOREIRA et al., 2008, p. 7,8).

Para estos autores, los NGP se inscriben en una difícil tensión entre las restricciones externas que América Latina tiene en el escenario global, dinamizado por la circulación financiera, y las decisiones políticas que los gobiernos asumen. Además, el rol subordinado y dependiente de América Latina 'obliga' a los gobiernos a mantener una política macroeconómica tendiente a la ortodoxia monetaria al tiempo que buscan desarrollar políticas que promuevan desarrollo estructural con cierto margen de autonomía interna y una búsqueda a nuevos mercados externos. 
Es en este mapa general, como respuesta a la crisis de la educación media y la preocupación por la inclusión socioeducativa, que se promueven programas de inclusión socioeducativa para intentar afrontar la desigualdad de los sectores pobres.

Mancebo y Monteiro (2009) analizaron esta situación en el marco de los procesos de segregación residencial que se han producido por el desplazamiento de la población de menores recursos a la periferia, contribuyendo al armado de los 'cinturones de pobreza'. Esto produjo la homogeneización socioeconómica de los barrios, de modo que "[...] la tónica fuertemente integradora de grupos con nivel socioeconómico diverso que Montevideo supo exhibir en el pasado como una característica distintiva, se ha quebrado en las últimas décadas" (MANCEBO; MONTEIRO, 2009, p. 22). Esto evidencia procesos de fragmentación social y escolar y se generan circuitos diferenciales en los procesos de distribución cultural. Este proceso llevó a que "a nivel del capital social colectivo suele resentirse la participación de los padres de clase media en la educación pública, habida cuenta que envían a sus hijos a colegios privados" (KAZTMAN en MANCEBO; MONTEIRO, 2009, p. 22), viéndose afectada de esta forma la integración social y la construcción de la ciudadanía democrática. Este proceso de segmentación educativa a nivel territorial condujo a un aumento de la matrícula privada en los barrios de mayor nivel socioeconómico.

Como desarrollamos en el apartado anterior, a estos procesos de segregación se suma el debilitamiento en la valoración de lo público asociado a la educación que se articula en los años 1990. En este sentido, se construye la idea de que en Uruguay existe una mala educación pública y una privada de "calidad".

Los aspectos mencionados y los elementos que dan cuenta de una "crisis educativa" han habilitado no solo cambios desde el sistema educativo público, sino que también la gestión privada se ha constituido en una aparente alternativa, promoviéndose estas modalidades de gestión público-privada.

\section{Presentación de las experiencias educativas vinculadas a las formas de privatización en el campo educativo}

\section{Programa de Aulas Comunitarias}

El Programa de Aulas Comunitarias (PAC) surge en el marco de los programas de inclusión educativa en el primer gobierno del FA, y se presenta como una política orientada a estudiantes desafiliados de Ciclo Básico con el objetivo de generar un "puente" con el sistema educativo formal. Participan del programa: jóvenes que han desertado del ciclo básico, adolescentes que están en riesgo de deserción y jóvenes que nunca cursaron el ciclo básico. Fue creado en el marco del Plan de Equidad, orientación general de la política social del FA, en el período del 2005 hasta 2010. 
Dicha propuesta nace con la intervención conjunta del Consejo de Educación Secundaria (CES), de la Administración Nacional de Educación Pública (ANEP) e Infamilia del Ministerio de Desarrollo Social. Una característica distintiva es que, a diferencia de otros programas de inclusión educativa implementados en el marco del "paradigma inclusivo" (MANCEBO; GOYENECHE, 2010) que fundamenta las estrategias de intervención diseñadas por el Estado desde el ańo 2005, el PAC tiene un modelo de cogestión entre el Estado y las Organizaciones de la Sociedad Civil (OSC). Como señalan Mancebo y Monteiro (2009), la idea de integrar las OSC en la provisión del servicio educativo se basó en: la necesidad de complementar los saberes de la educación formal con los de la no formal; la personalización en el trato a los estudiantes y el impulso al protagonismo adolescente, que serían favorecidos en aulas comunitarias debido a su pequeña escala; y la posibilidad de potenciar el PAC en base al conocimiento de la comunidad y el trabajo en red que cada OSC ha acumulado a partir del desarrollo de otros proyectos sociales.

De este modo, el programa se identifica por tener una institucionalidad particular, caracterizada por la intersectorialidad estatal (CES e Infamilia) y la cogestión del Estado con las OSC. En este marco, el CES tiene la responsabilidad técnico-pedagógica de: designar, supervisar y remunerar a los docentes que dictan las asignaturas y los profesores referentes. El Programa Infamilia, como contraparte, paga durante un año la contratación de las OSC que gestionan las aulas, pasando posteriormente el financiamiento al CES, además de contribuir con el monitoreo y la evaluación del programa. Las OSC contratadas aportan el espacio físico para el aula comunitaria, gestionan el aula a través de las figuras del coordinador y los técnicos, y además desarrollan talleres temáticos en función de su trayectoria de trabajo socioeducativo con adolescentes que provienen de sectores en situación de vulnerabilidad. A nivel del territorio, cada aula comunitaria cuenta con un equipo socioeducativo.

En síntesis, esta modalidad se podría caracterizar como mixta en tanto se desarrolla en el marco de una co-responsabilidad entre el Estado (CES - Infamilia) y las OSC. Los equipos docentes, los currículos y las actividades también son mixtos en tanto sostienen los elementos centrales del curriculum universal del ciclo básico e integran, en forma situada, otras actividades.

\section{Liceos Jubilar e Impulso: dos modelos de gestión privada con financiamiento público}

A diferencia del PAC, los liceos Jubilar e Impulso se inscriben en una modalidad de gestión privada con financiamiento público, que son característicos de las formas de privatización en el campo educativo. 
Ambos se ubican en un sector catalogado como vulnerable en Uruguay, más precisamente en la Cuenca Casavalle. Algunas de las problemáticas a las que aluden estas propuestas son: clases superpobladas y riesgos de abandono por múltiples causas como: trabajo infantil y adolescente, falta de seguridad, distancia con los otros liceos (que mencionan no es solo de kilómetros sino que también refieren a aspectos culturales) o faltas por razones familiares.

El liceo “Jubilar Juan Pablo II” surgió en el año 2002, presentándose como el primer liceo gratuito de gestión privada y católico, que ofrece educación media básica. Con respecto al modelo organizacional, constituye un centro educativo de tiempo completo de nueve horas con cursos reglamentarios exigidos por el CES y varios talleres educativos y recreativos. Es financiado casi exclusivamente con donaciones privadas, si bien se agrega una cuota familiar dentro de las posibilidades.

Los resultados educativos revelan que en los últimos años no han habido deserciones y las tasas de repetición no alcanzan al 2\% de los estudiantes, cifras destacables cuando se analizan en el contexto de una tasa de repetición promedio del 40\% en el ciclo básico en los liceos de Montevideo (MEC, 2009).

Los criterios de selección del alumnado son estrictos; los aspirantes deben vivir en los barrios de la Cuenca de Casavalle; no deben tener más de dos años de desfasaje con respecto al nivel al que aspiran y el nivel familiar debe estar por debajo de la línea de pobreza. De acuerdo a los datos que difunden, el liceo Jubilar tiene una lista de aspirantes mayor a la de los cupos que puede cubrir por sorteo.

Por su parte, el liceo Impulso comienza a funcionar en el año 2013 con el apoyo de empresas privadas. Es el primer liceo de tiempo completo aconfesional y gratuito de gestión privada que apunta a contribuir con la disminución de la "brecha de aprendizaje de alumnos de contexto crítico". La selección de los estudiantes también es por sorteo. Esta institución procura un "modelo educativo por excelencia", haciendo referencia a los resultados educativos, con cero caso de repetición. En ambos liceos se establecen estrictos criterios de selección de los docentes y el personal no docente.

En síntesis, los dos liceos descritos se inscriben en una lógica diferente al PAC, adoptándose una modalidad de gestión privada con financiamiento público propia de los procesos privatizadores. Asimismo, es pertinente señalar que la información que estas instituciones proporcionan es escasa. En ambas instituciones, existen ciertos rasgos comunes: los criterios de selección del estudiantado y del personal docente; la extensión del tiempo pedagógico; el seguimiento personalizado de los estudiantes y la instrumentación de tutorías. Estos elementos, claramente, podrían explicar los buenos resultados educativos que exhiben. En este sentido, es necesario subrayar que los mismos no 
se vinculan con modalidades de gestión sino con elementos de un diseño de enseñanza.

Finalmente, interesa enfatizar, en términos generales, que en nuestro país la mayoría de estas iniciativas de modelos de gestión público-privado en enseñanza media surgen en el seno de los NGP que, con diferente entonación, se han caracterizado por un rechazo al modelo neoliberal de los 1990. En las líneas trabajadas en este artículo, esto podría entenderse en el marco de la construcción de los universos de sentido en los cuales lo público ha sufrido un progresivo proceso de desvalorización que trasciende los enclaves político - partidarios buscando un asiento en elementos "del sentido común".

\section{Conclusiones}

Fueron analizados tres modelos de gestión público-privado desarrollados en la enseńanza media en las últimas décadas en Uruguay, en el marco de la disputa en torno al papel que el Estado, las OSC y las empresas ocupan en el ámbito de la educación pública. Estos ejemplos presentan rasgos similares a los desarrollados en otros países de la región, no obstante asumen las particularidades de la cultura política uruguaya, el carácter gradualista que se conjuga con una racionalidad argumentativa que promueve el equilibrio y busca establecer estrategias de consenso.

Desde una perspectiva que recupera la historicidad a los efectos de analizar estas experiencias en la trama de las configuraciones discursivas que les dan sentido, hemos visualizado que, en el contexto de surgimiento de la escuela moderna, la educación constituía uno de los pilares para construir una identidad nacional y de integración social. La escuela surgió como espacio simbólico cuyo propósito era difundir un ideario igualitarista e integrador. La igualdad se presentó como principio (utópico) y valor democratizador del proyecto educativo moderno, si bien se traducía en un opacamiento de la diversidad.

En la década de los noventa, las configuraciones de sentido en torno a lo escolar sufren alteraciones. $\mathrm{Al}$ respecto, se articula un discurso que reconoce las dificultades del sistema educativo para dar respuesta a aquellos sectores que históricamente fueron postergados ubicando como "responsable" al Estado. En este contexto, la "crisis educativa", particularmente de la educación media, se aprecia en las dificultades de retención y aprendizajes de todos los sujetos. La educación como discurso igualador e igualitario pierde potencialidad. Las políticas de los 1990 implementaron programas focalizados y asistenciales al tiempo que contribuyeron con la desvalorización de lo universal y lo público y habilitaron el progresivo encanto por la educación privada. Esta valoración por lo privado se 
instalará en el imaginario social junto al descrédito de la educación pública la cual, paulatinamente, se visualizará como educación de los pobres.

Desde otra arista, a partir de la asunción de los NGP (FA), surgieron programas focalizados fundamentados en el paradigma de la inclusión con el fin de afrontar los problemas de desigualdad en el marco de los procesos de segregación residencial agudizados en las décadas anteriores.

Los procesos de segregación y segmentación persistentes junto a la crítica de lo público que fue articulada en los años 1990 y la revaloración por lo privado han ido configurando los nuevos discursos que presentan a las modalidades de gestión público-privada como las alternativas. El PAC es un modelo mixto del Estado y las OSC que impulsa una política de inclusión focalizada y los liceos Jubilar e Impulso se desarrollan con una modalidad de gestión privada con financiamiento público. Ambas modalidades constituyen formas diferentes y graduales (propias de la cultura uruguaya) de articulación público-privado a nivel educativo, que procuran un asiento argumentativo y racional en los diagnósticos en torno a la crisis. Estas modalidades reflejan, en la materialidad histórica, la disputa en el campo simbólico por sentidos en torno a lo público.

En esta disputa, en el contexto actual, conviven en forma conflictiva discursividades caracterizadas por el desvalor de lo público y el progresivo encanto de la "eficiencia" privada con aquellas que continúan reivindicando el valor de lo público y el rol central del Estado como forma de garantizar el derecho a la educación y la igualdad.

En esta línea, desde una orientación de justicia, entendemos que buscar alternativas privatizadoras implica continuar reforzando y promoviendo lógicas de segmentación del sistema educativo, profundizando los circuitos de educación paralelos. Ante esta situación nos interrogamos: ¿̨no es tiempo de repensar la forma escolar en su globalidad? En este sentido, se torna un desafío analizar las políticas focalizadas desde una vocación universal e igualitaria. Ello constituye una apuesta por todos los sujetos y una defensa de la escuela pública como espacio simbólico y material que instituye un "nosotros". Supone, en otras palabras, una apuesta por la justicia. En este sentido:

\footnotetext{
Quizás es la pregunta por la justicia la que está faltando en el debate sobre la diversidad en la educación. Se trata de un interrogante político y ético que atraviesa al conjunto de la organización escolar y al curriculum, y no se resuelve en el espacio de la 'educación para los pobres' sino que exige replantear el horizonte de igualdad ciudadana que estamos proponiendo a las nuevas generaciones, e involucra al sistema en su conjunto. (DUSSEL, 2004).
}

Destacamos en esta apuesta el carácter político de la educación, en el marco de la cual el Estado frente a los problemas de injusticia/desigualdad entre instituciones 
y sujetos ocupa un papel central. En este posicionamiento de defensa de lo público, se deben analizar las operaciones ideológicas en el marco de las cuales los discursos construyen sentidos en torno al "valor" que las nuevas maneras privatizadoras de la educación tendrían. Estas operaciones discursivas opacan las causas estructurales de las desigualdades e injusticias y presentan a las desigualdades como "naturales".

Reposicionar a la Escuela como espacio y asunto público y recuperar la proclama igualitarista característica del surgimiento de la escuela moderna resignificándola en el contexto actual, en el marco del cual el respeto a la diferencia - que no debe ser traducida en desigualdad - se conjugue con el valor de lo común, se tornan objetivos centrales. No se trata de desentenderse de los problemas institucionales sino de integrarlos en una dimensión política. De este modo,

[...] asumir un proyecto de educación pública supone pensar los modos de articular iniciativas y responsabilidades del Estado con aquellas de las instituciones, enmarcadas en el principio de "justo para todos" y no sólo "bueno para nosotros". (FRIGERIO, 2000, p. 13).

Desde este lugar, se trata de concebir la escuela como un asunto político integrado en un proyecto de formación de los ciudadanos democráticos.

\section{Notas}

1. José Pedro Varela fue el impulsor del proceso de la reforma educativa en Uruguay en el segundo tercio del siglo XIX. Como Director de Instrucción Pública de la Junta Económica Administrativa, inició en 1876 el proceso de reforma bajo los principios de gratuidad, laicidad y obligatoriedad.

2. En Uruguay, la dictadura cívico-militar se extendió desde el 27 de junio de 1973 hasta el 1º de marzo de 1985 en que el doctor Julio María Sanguinetti asume la presidencia.

3. La educación básica pública en Uruguay se inscribe en la ANEP, la cual es un ente autónomo sin dependencia jerárquica con el Ministerio de Educación y Cultura. Esta se organiza en ciclos de educación: inicial de tres años; primaria de seis años; media básica de tres años y media superior de tres años. Históricamente, la educación media, particularmente la media superior, tuvo un carácter selectivo y elitista.

4. En Uruguay, las características de los cambios en la administración central tuvieron tres líneas estratégicas: creciente transferencia de las funciones del Estado a la sociedad civil; reconstrucción de un núcleo central en la administración para coordinar las nuevas formas de prestación de servicios públicos; y desarrollo de un sistema de control por resultados (NARBONDO; RAMOS, 2004). No obstante, el desarrollo de estas transformaciones varió en diversos sectores en función del poder relativo de los actores sociales de cada sector, capacidad de los técnicos y de los dispositivos estratégicos empleados para la implementación de las reformas, etc. (FILGUEIRA; MORAES, 2000; BENTANCUR, 2008). 
5. En 1992, el 79\% de la población uruguaya, por medio de un plebiscito promovido por las organizaciones sociales, deroga la Ley de Empresas Públicas votada en el gobierno del Partido Nacional con el apoyo del Partido Colorado y la oposición del FA. Esta manifestación democrática significó un freno al proceso privatizador que la coalición de los dos partidos tradicionales del Uruguay impulsaban.

6. América Latina, paulatinamente, se fue modificando con la asunción de gobiernos más próximos a posiciones de centro-izquierda que tienen en común un discurso de oposición al Consenso de Washington y las reformas neoliberales emanadas del mismo, así como la búsqueda de articulaciones políticas distintas a nivel nacional, regional e internacional. No obstante estos aspectos generales y comunes, los diversos gobiernos, tienen sus particularidades y rasgos diferenciales.

\section{Referencias}

ARISTIMUÑO, A. El abandono de los estudios del nivel medio en Uruguay: un problema complejo y persistente. Revista Iberoamericana sobre Calidad, Eficacia y Cambio en Educación, v. 7, n. 4, p. 180-197, 2009. Disponible en: <http://www. rinace.net/reice/numeros/arts/vol7num4/art9.htm>. Acceso el: 8 nov. 2015.

BENTANCUR, N. Las reformas educativas de los años noventa en Argentina, Chile y Uruguay. Racionalidad política, impactos y legados para la agenda actual. Montevideo: Banda Oriental, 2008.

BORDOLI, E.; MARTINIS, P. Relaciones entre educación y pobreza. Continuidades y rupturas del discurso moderno. In: SERNA, M. (Coord.). Pobreza y (des)igualdad en Uruguay: una relación en debate. Montevideo: F.C.S., 2010, p. 228-240.

BORDOLI, E. El olvido de la igualdad en el nuevo discurso educativo. In: MARTINIS, P.; REDONDO, P. (Comps.) Igualdad y educación. Escrituras entre dos orillas. Buenos Aires: Del Estante Editorial, 2006, p. 185-2004.

CASASSUS, J. Concertación y alianzas en educación. In: VIAL CORREA, G. (Coord.) ¿Es posible concertar las politicas educativas? Buenos Aires: Facultad Latinoamericana de Ciencias Sociales, Miño y Dávila, 1995.

DUSSEL, I. La escuela y la diversidad: un debate necesario. 2004. Disponible en: <http://ipes.anep.edu.uy/documentos/curso dir 07/materiales/escuela uno.pdf $>$. Acceso el: 8 nov. 2015.

FERNÁNDEZ, T. Balance de las politicas de inclusión educativa en la Educación Media de Uruguay (2005-2009). Ponencia presentada en la Mesa: "Políticas de inclusión educativa" en las IX Jornadas de la Facultad de Ciencias Sociales de la UdelaR, Montevideo, 2010. Disponible en: <http://cienciassociales.edu.uy/wp-content/uploads/2013/archivos/ Mesa 12 y 17 FERNANDEZ.pdf>. Acceso el: 12 oct. 2014.

FILGUEIRA, F.; MORAES, J.A. Contexto y estrategias de las reformas institucionales en la seguridad social, la educación y la salud en Uruguay". Revista Uruguaya de Ciencias Politicas, n. 12, Montevideo, 2000. 
FRIGERIO, G. ¿Las reformas educativas reforman las escuelas o las escuelas reforman las reformas? Chile: UNESCO, 2000.

GENTILI, P.; STUBRIN, F. Presentación. In: GENTILI, P. et al. (Org.), Políticas de privatización, espacio público y educación en América Latina. Rosario / Santa Fe: HomoSapiens, CLCSO, 2009, p. 9-14.

GIROUX, H. La educación y la crisis del valor de lo público. Montevideo: Criatura, 2012.

LANZARO, J. La reforma educativa en Uruguay (1995-2000): virtudes y problemas de una iniciativa heterodoxa, Serie Política Social No 91. Santiago de Chile: CEPAL, 2004, p. $1-44$.

LEHER, R. Estrategias de mercantilización de la educación y tiempos desiguales de los tratados de libre comercio: el caso de Brasil. In: GENTILI, P. et al. (Org.), Políticas de privatización, espacio público y educación en América Latina. Rosario / Santa Fe: HomoSapiens, CLCSO, 2009, p. 15-66.

MANCEBO, M.E.; GOYENECHE, G. Las politicas de inclusión educativa: entre la exclusión social y la innovación pedagógica. Ponencia presentada en la Mesa "Políticas de inclusión educativa” en las IX Jornadas de la Facultad de Ciencias Sociales de la UdelaR, Montevideo, 2010. Disponible en: <http://cienciassociales.edu.uy/wpcontent/uploads/2013/archivos/Mesa_12_y_17_Mancebo-Goyeneche.pdf>. Acceso el: 12 oct. 2014.

MANCEBO, M.E.; MONTEIRO, L. El Programa de Aulas Comunitarias de Uruguay: Un Puente hacia la Inclusión en la Educación Media. REICE. Revista Iberoamericana sobre Calidad, Eficacia y Cambio en Educación, v.7, n. 4, 2009, p. 277-291. Disponible en: <http://www.rinace.net/reice/numeros/arts/vol7num4/art14.pdf >. Acceso el: 24 nov. 2010.

MARTINIS, P. Educación, pobreza e igualdad: del "niño carente" al "sujeto de la educación”. In: MARTINIS, P.; REDONDO, P. (Comps.), Igualdad y educación. Escrituras entre dos orillas. Buenos Aires: Del Estante Editorial, 2006, p. 13-32.

- Educación, pobreza y seguridad en el Uruguay de la década de los noventa. Montevideo: CSIC - UdelaR, 2013.

MAYORGA, F. El proyecto político del MAS: ¿hacia la construcción de un gobierno mayoritario? In: AIBAR, J.Y.; VÁZQUEZ, D. (Coord.). ¿Autoritarismo o democracia? Hugo Chávez y Evo Morales. México: FLACSO, 2009, p. 19-58.

MINISTERIO DE EDUCACIÓN Y CULTURA (MEC). Anuario Estadístico de Educación. 2009. Disponible en: <http://www.mec.gub.uy/innovaportal/file/4282/1/ anuario estadistico educacion 2009.pdf >. Acceso el: 20 nov. 2015.

MOREIRA, C. La reforma de Estado en Uruguay: cuestionando el gradualismo y la heterodoxia. In: CALAMÉ, P.Y.; TALMANT, A. (Comps.), Con el Estado en el corazón. El andamiaje de la gobernancia. Montevideo: Trilce, 2001.

MOREIRA, C. et al. Introducción. In: MOREIRA, C. et al. (Org.), La nueva politica en América Latina. Rupturas y continuidades. Montevideo: Trilce, 2008, p. 7-22. 
NARBONDO, P.; RAMOS, C. Reforma administrativa y capacidad estatal de conducción. In: CALAMÉ, P.; TALMANT, A. (Comps.) Con el estado en el corazón. El andamiaje de la gobernancia. Montevideo: Trilce, 2004.

PÊCHEUX, M. O discurso. Estructura ou acontecimento. Campinas: Pontes, 1990.

PINEAU, P. et al. La escuela como máquina de educar. Tres escritos sobre un proyecto de la modernidad. Buenos Aires: Paidós, 2001.

VARELA, J.P. La educación del Pueblo. Montevideo: Cuaderno LEA, 1973.

Recibido en 16 de diciembre de 2015.

Aprobado en 29 de enero de 2016. 